\title{
ELECTRONIC RECORDING OF TRAFFIC INCIDENTS
}

doi:10.1136/injuryprev-2012-040580e.20

${ }^{1} \mathrm{~J}$ Ahn*, ${ }^{1} \mathrm{R}$ Leamy, ${ }^{3} \mathrm{Y}$ Liu, ${ }^{3} \mathrm{~S}$ Manoharan, ${ }^{2} \mathrm{M}$ Stables, ${ }^{3} \mathrm{~S}$ Zheng. ${ }^{1}$ New Zealand Transport Agency, Wellington, New Zealand; ${ }^{2}$ New Zealand Police, Wellington, New Zealand; ${ }^{3}$ University of Auckland, Auckland, New Zealand

Background Traffic crash reports (TCRs) are the forms completed by police officers at the scene of road crashes. They record the 
details of when, how and why the crash happened. The reports are used, among other things, to mitigate accidents of similar nature and to build a safer travel environment.

Aims/Objectives/Purpose TCRs in New Zealand are paper forms. Manual entries on paper forms have several drawbacks including entry errors and illegibility. To analyse the data collected through paper forms, the data need to be re-entered into an electronic format. Data consistency checks are not possible in manual data entry: for instance, a single response to a single response question cannot be enforced. This paper presents a framework for a mobile application that aims to replace the paper form.

Methods The paper develops a mobile framework with the following key aspects: there is no need for dual data entry; consistency checks are enforced; some of the information is auto-filled (eg, time and location); some data are auto-captured (eg, driver information from the bar-code of the driving license); some media can be attached (eg, photos, sketches, and audio). The data so collected is transferred easily to the processing centre. This transfer is in a manner that lends itself to easy processing.

Results/Outcomes There are two distinct user-interface styles: a form-based one that translates the current paper form to a comparable electronic format; and the second 1 is incident-focused. The incident-focused style is radically different but is more intuitive and offers a better user experience. With both styles, collected data is persisted as XML conforming to a standardised schema.

Significance/Contribution to the Field The proposed framework (a) eases the completion of the report (eg, auto-filling and autocapture features) and (b) eases the end-analysis of the collected data. The usability of the user-interface is deemed high. 